\title{
Diamantane Suspended Single Copper Atoms
}

\author{
Hong-Ying Gao, ${ }^{*, \infty, \ddagger, \nabla}$ Marina Šekutor, ${ }^{\S, \|}$ Lacheng Liu, ${ }^{\infty, \ddagger}$ Alexander Timmer, ${ }^{\infty, \ddagger}$ Hannah Schreyer, ${ }^{\perp}$ \\ Harry Mönig, ${ }^{\infty, \ddagger}$ Saeed Amirjalayer, ${ }^{\infty, \ddagger}$ Natalie A. Fokina, ${ }^{\S}$ Armido Studer, ${ }^{\#}$ Peter R. Schreiner, ${ }^{*}, \S$ \\ and Harald Fuchs $*, \infty, \ddagger$ \\ ${ }^{\infty}$ Center for Nanotechnology, Heisenberg Straße 11, Münster 48149, Germany \\ ${ }^{\ddagger}$ Department of Physics, Munster University, Wilhelm-Klemm-Straße 10, Münster 48149, Germany \\ ${ }^{\S}$ Institute of Organic Chemistry, Justus Liebig University, Heinrich-Buff-Ring 17, and Center for Materials Research (LaMa), Justus \\ Liebig University, Heinrich-Buff-Ring 16, Giessen 35392, Germany \\ "Department of Organic Chemistry and Biochemistry, Ruđer Bošković Institute, Bijenička cesta 54, Zagreb 10 000, Croatia \\ ${ }^{\perp}$ Max-Planck-Institut für Kohlenforschung, Kaiser-Wilhelm-Platz 1, Mülheim an der Ruhr 45470, Germany \\ \#Institute of Organic Chemistry, Münster University, Correns Straße 40, Münster 48149, Germany \\ ${ }^{\nabla}$ School of Chemical Engineering and Technology, Tianjin University, Tianjin 300072, China
}

\begin{abstract}
Single chains of metal atoms are expected to be perfect onedimensional nanowires in nanotechnology, due to their quantum nature including tunable electronic or spin coupling strengths. However, it is still rather difficult to fabricate such nanowires with metallic atoms under directional and separation control. Here, we succeeded in building higherorder single diamondoid-chains from the lower-order chains using a chemically well-controlled approach that employs diamondoids on metal surfaces. This approach results in higher-order diamondoid double chains by linking two neighboring single chains, and ultimately forms a central chain consisting of single $\mathrm{Cu}$ atoms suspended by the diamantane framework. The suspended $\mathrm{Cu}$ atoms are placed above the metal surface with a periodic distance of $0.67 \pm 0.01 \mathrm{~nm}$. Our bottom-up approach will allow detailed experimental investigations of the properties of these exciting suspended

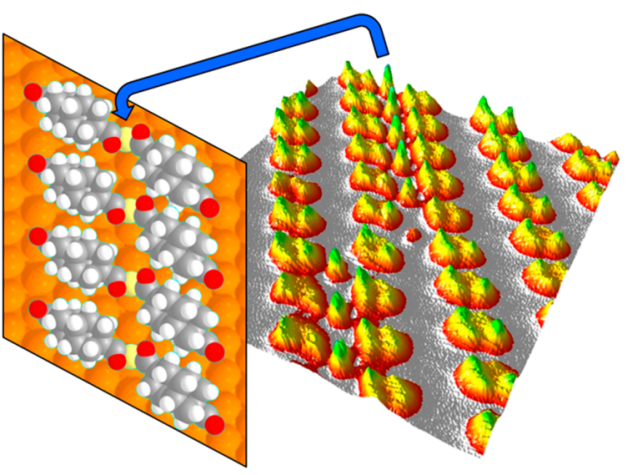
metal atoms (for example, quantized conductance, spin coupling, as well as transfer, etc.). Furthermore, we also identified different spatial configurations on the metal surfaces in on-surface reaction processes using high-resolution AFM imaging and density functional theory computations. Our findings broaden the on-surface synthesis concept from $2 \mathrm{D}$ planar aromatic molecules to 3D bulky aliphatic molecules.
\end{abstract}

\section{INTRODUCTION}

As we are quickly approaching the size limit of silicon-based technologies, further miniaturization is only possible by moving toward the ultimate limit of single molecule (or even single atom) building blocks as key components in electronic circuitry. This emerging field termed molecular-scale electronics or single-molecule electronics relies on the premise that precise control of nanometer distances is possible and that the required molecular building blocks can be produced precisely, reproducibly, and on a large scale. ${ }^{1,2}$ Despite many challenges facing the application of miniaturized electronic devices to real life systems, nanoscale devices and elaborated principles of their application already exist today, some examples being systems capable of charge transport across molecular monolayers, ${ }^{3}$ organic spintronics, ${ }^{4,5}$ single-atom catalysts, etc. ${ }^{6,7}$ Additionally, molecular junctions where one organic molecule acts as a bridging unit and connects neighboring metal atoms are illustrative examples of unimolecular rectifiers, devices capable of current conductance only in one direction. ${ }^{8-10}$

Another course to take when designing nanomolecular architectures is the manufacture of nanowires, scaffolds capable of reliable conductance at long distances, ${ }^{11}$ while retaining desired device properties emerging from their inherent quantum nature. ${ }^{12}$ An important early study of nanodevice electron transport showed that single chains of $\mathrm{Au}$ atoms display quantized conductance, meaning that they act as onedimensional quantized nanowires. ${ }^{13,14}$ Such noble metal chains are therefore expected to be near perfect one-dimensional conductors. While research on gold chains has since been continued, ${ }^{15,16}$ copper has been out of the spotlight for quite some time. ${ }^{17,18}$ Despite continuous efforts to perfect the methods for generating ultrathin nanowires a few $\mathrm{Cu}$ atoms wide, ${ }^{19-22}$ a single atom width nanowire (potential conductor) 
is still the ultimate dream that could also be accessible by a single molecule approach. In such chains, directional control and separation of conductive and semiconductor moieties without the use of conventional passive separation layers could be possible. Moreover, by delving into the quantum nature of the atom-wide nanowires, exciting properties such as tunable electronic or spin coupling strengths could become accessible.

Like in so many areas of nanotechnology, metallic nanostructures of interest can be designed using either a top down or a bottom up approach with the same final goal of precise shape control that provides the necessary selectivity and functionality. ${ }^{23}$ Our research in this arena follows the bottom up principle as a way to avoid randomness of the formed ensembles, and to this end we used diamondoids, ${ }^{24}$ naturally occurring $^{25}$ nanoscale diamond-like hydrocarbons capable of precise self-assembly due to their controllable functionalization $^{26}$ and stabilization of their monolayers by London dispersion interactions. ${ }^{27}$ Some recent examples of diamondoid scaffold applicability in single-molecule electronics include diamondoid monolayers capable of monochromatic electron photoemission, ${ }^{28}$ linear-chain nanodiamond wires inside carbon nanotubes, ${ }^{29,30}$ diamondoid functionalized $\mathrm{Au}-$

coated Ge nanowire field emitters, ${ }^{31}$ and a fullerenediamantane unimolecular rectifier ${ }^{10}$ In the context of our present study, an especially notable example of applicable nanowires is a hybrid metal-diamondoid chalcogenide

material prepared recently. ${ }^{32}$ More specifically, the metalorganic chalcogenide (MOC) consisted of an electrically conductive inorganic core that retained the band-like transport properties and a diamondoid outer part that acted both as a molecular structure-directing agent and as an insulator. The observed nanowire growth was directed by diamondoid selfassembly, producing a strongly confined conducting material.

Now we turn our attention to chain formation on metal surfaces with the hope of producing uniform single-atom wide metal chains that are held in place by diamondoid side anchors. We chose copper as the metal because $\mathrm{Cu}$ nanowires would be highly desirable for a variety of applications. We employed modern scanning tunneling microscopy (STM) and atomic force microscopy (AFM) techniques to characterize the selfassembled structures in combination with bis-apical 4,9diamantane dicarboxylic acid (DDA) ${ }^{33}$ as the precursor for the diamondoid directed self-assembly; carboxylic acids readily undergo annealing on metal surfaces. ${ }^{34}$ To generate the diamantane anchor assemblies, we used the tools of on-surface synthesis ${ }^{35}$ that we previously successfully applied when performing surface reactions such as decarboxylative coupling, ${ }^{34}$ peroxide coupling, ${ }^{36}$ and $\sigma$-bond metathesis ${ }^{37}$ that require precise tuning of the reaction conditions.

As illustrated in Figure 1, here we present a chemically controlled approach to anchor DDA diamondoids at the metal surface, building of higher-order single diamondoid-chains from the lower-order ones, generation of higher-order diamondoid double chains by linking two neighboring single chains, and ultimately the formation of a central metallic chain consisting of single $\mathrm{Cu}$ atoms anchored by the diamantane framework. We expect this bottom up strategy to provide a route to a class of one-dimensional suspended metallic atom chains. Furthermore, we also successfully identified the different DDA geometries in on-surface reactions by high-resolution AFM imaging and density functional (DFT) computations. This study is likely to take the on-surface
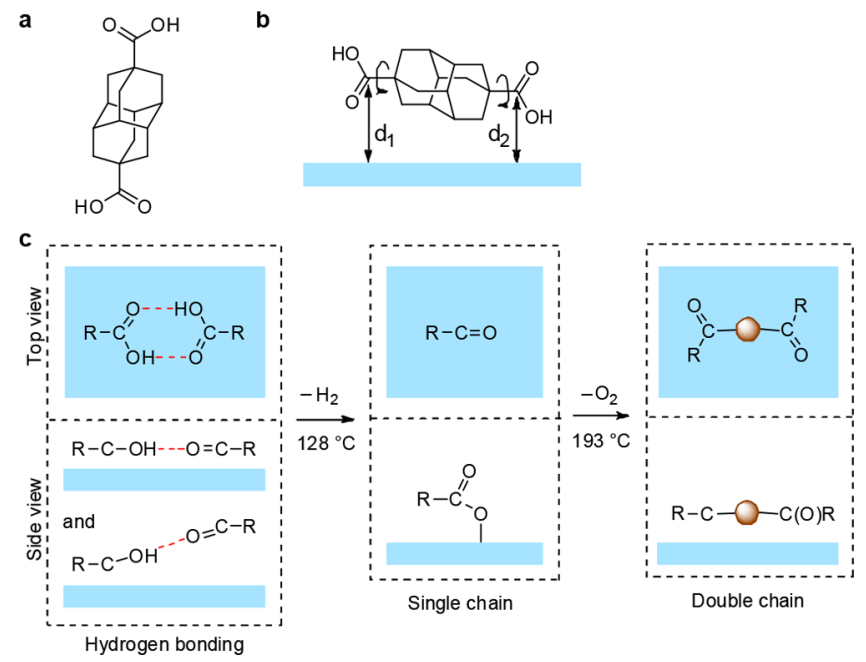

Figure 1. On-surface reactions of 4,9-diamantane dicarboxylic acid (DDA). ( $a$ and b) Structure of DDA and its proposed placement on the $\mathrm{Cu}(111)$ surface. (c) Proposed reaction pathway for stepwise dehydrogenation and deoxygenation of spatial DDA at metal surface.

synthesis concept from 2D planar aromatic to 3D bulky aliphatic molecules.

\section{EXPERIMENTAL SECTION}

STM Measurements. The initial STM experiments were performed by an Omicron low temperature $(78 \mathrm{~K})$ scanning tunneling microscope (LT-STM) with a base pressure of $1 \times 10^{-10} \mathrm{mbar}$. STM images were acquired in constant current mode, with the sample biased. $\mathrm{Au}(111), \mathrm{Ag}(111)$, and $\mathrm{Cu}(111)$ surfaces were cleaned by several cycles of sputtering and annealing. 4,9-Diamantane dicarboxylic acid (DDA) $)^{33}$ was evaporated from a crucible at $190{ }^{\circ} \mathrm{C}$ with a deposition speed of $\sim 0.067 \mathrm{~mL} / \mathrm{min}$ (calibrated by STM images). Thermal annealing toward the samples was monitored by an IR detector.

nc-AFM and XPS Measurements. The nc-AFM measurements were conducted with an Omicron low-temperature $(\sim 5 \mathrm{~K})$ scanning probe microscope (LT-STM/AFM) with a base pressure of $1 \times 10^{-10}$ mbar. A tuning fork-based cantilever in the qPlus configuration, an etched $\mathrm{W}$-tip in the frequency modulation mode, was used. The qPlus sensor parameters were: spring constant $k \approx 2000 \mathrm{~N} \mathrm{~m}^{-1}$, resonance frequency $f_{0} \approx 24 \mathrm{kHz}$, and typical quality factors were in the range $Q$ $=20$ 000-40 000 (at liquid He temperature). All AFM images were acquired at constant height with an oxygen-terminated copper tip. ${ }^{38,39}$ As our nc-AFM scanning, we also recorded the simultaneous current image without any bias applied, defined AFM-current image. This LTSTM/AFM system was directly connected to an XPS analysis system, which allowed for in situ sample transfer. The base pressure of the XPS part was around $2 \times 10^{-10}$ mbar. Photoelectrons were excited by a monochromated $\mathrm{Al} \mathrm{K}_{\alpha} \mathrm{X}$-ray source and analyzed by a SPECS PHOIBOS 100 hemispherical analyzer in conjunction with a $2 \mathrm{D}$ delayline detector. Additionally, the molecular deposition parameters were established at $175{ }^{\circ} \mathrm{C}$, with a deposition rate $\sim 0.125 \mathrm{ML} / \mathrm{min}$ (calibrated by STM image). The XPS binding energy simulations were done by DFT (for details, see the Supporting Information).

Computations. Geometry optimizations of DDA and bisdehydrogenated DDA were performed at the B3LYP-D3(BJ)/6- 311 $+\mathrm{G}(\mathrm{d}, \mathrm{p})$ level of theory ${ }^{40-43}$ using Gaussian $16{ }^{44}$ without the $\mathrm{Cu}$ surface. Chem3D software was used to visualize the obtained geometric configuration of DDA and to measure the atom-to-atom distance, and the corresponding top hydrogen atoms were marked in green on the presented figures. Semiempirical computations of DDA molecules on a $\mathrm{Cu}(111)$ surface were performed using the GFN-xTB approach developed by Grimme et al. ${ }^{45}$ A copper crystal surface cutout consisting of $169 \mathrm{Cu}$ atoms (dimensions: $26 \times 15 \times 2.5 \AA$ ) or 234 
a

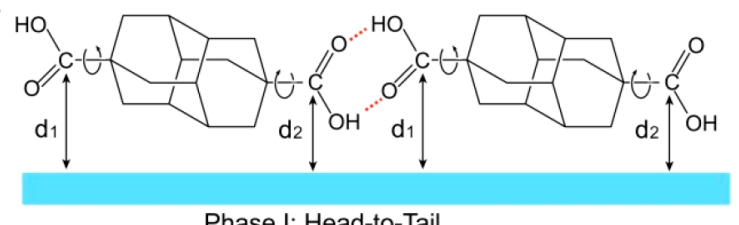

Phase I: Head-to-Tail

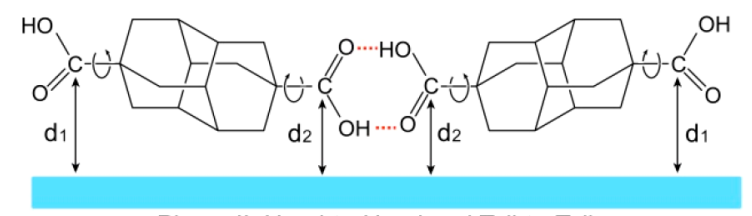

Phase II: Head-to-Head and Tail-to-Tail

b

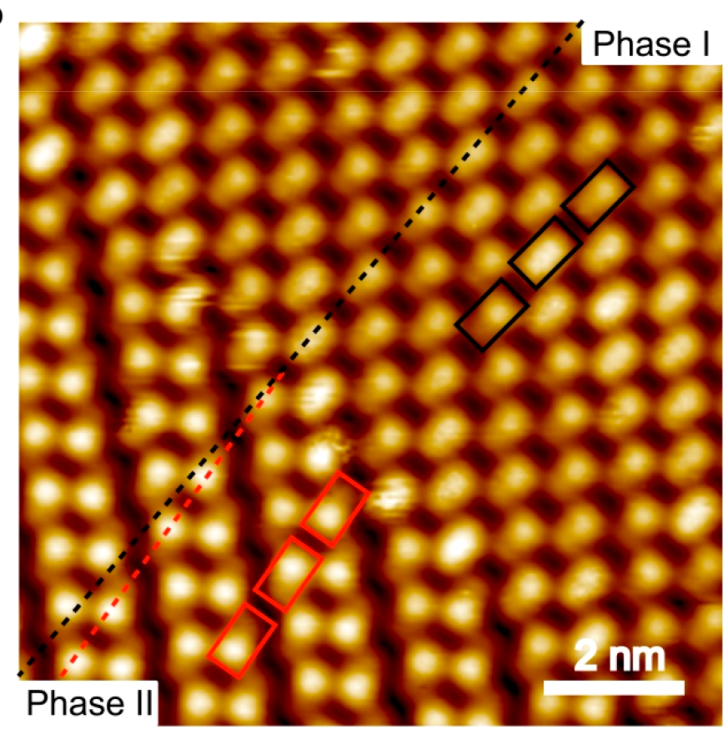

C i)
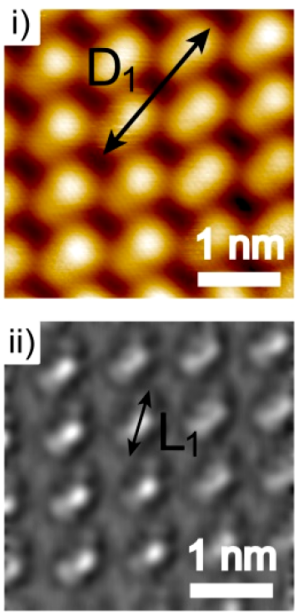

d
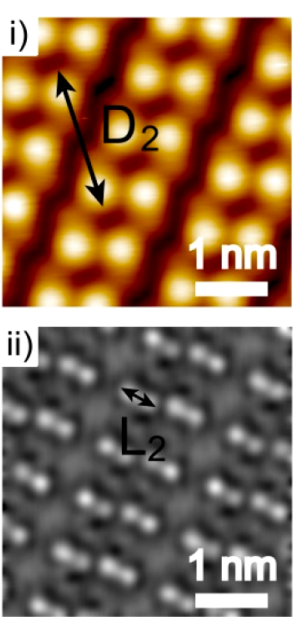

ii)
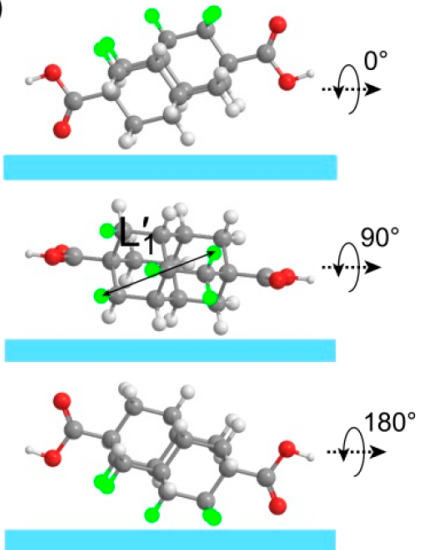

iii)
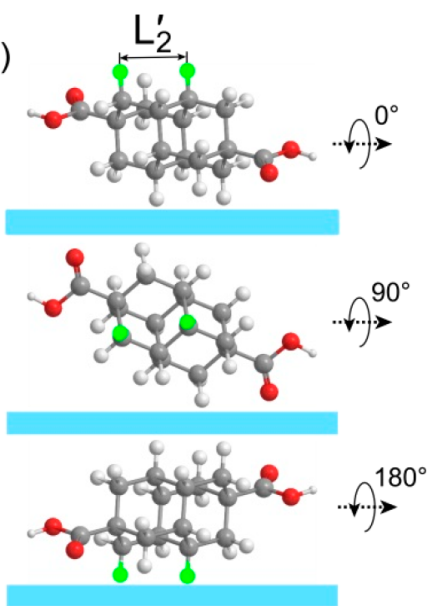

Figure 2. Self-assembled structure of DDA on a $\mathrm{Cu}(111)$ surface. (a) Proposed hydrogen-bond modes: head-to-tail (phase I) and head-to-head/ tail-to-tail (phase II). (b) STM image of the self-assembly showing both hydrogen-bonding phases I and II $(-2 \mathrm{~V}, 6 \mathrm{pA}, 10 \times 10 \mathrm{~nm})$. (c and d) Magnified STM images $(\mathrm{c}-\mathrm{i}, 3.5 \times 3.5 \mathrm{~nm}$ and d-i, $4 \times 4 \mathrm{~nm},-2 \mathrm{~V}, 6 \mathrm{pA})$ and nc-AFM constant height images $(\mathrm{c}-\mathrm{ii}, 3.5 \times 3.5 \mathrm{~nm}$ and d-ii, $4 \times 4$ $\mathrm{nm}$, oscillation amplitude $A_{\mathrm{osc}}=0.7 \AA, V=25 \mathrm{mV}$ ), as well as their proposed spatial configurations with top hydrogen atoms marked in green (c-iii and d-iii: top, the side view of DDA; middle, the side view of DDA after being rotated $90^{\circ}$ along the axis parallel to substrate surface; bottom, the side view of DDA after being rotated $180^{\circ}$ along the axis parallel to substrate surface).

$\mathrm{Cu}$ atoms (dimensions: $38 \times 15 \times 2.5 \AA$ ) was taken, and the atoms were frozen to simulate the copper lattice. DDA molecules were placed on the corresponding copper slab, optimized, and the obtained geometries compared to the structural parameters obtained from AFM imaging, as described previously. ${ }^{46}$ Further details and the corresponding coordinates are provided in the Supporting Information.

\section{RESULTS AND DISCUSSION}

Hydrogen-Bonding Self-Assembly of DDA. As we previously demonstrated, it is possible for diamondoids to adopt different orientations on metal surfaces due to their bulkiness, rigidity, and incompressible $3 \mathrm{D}$ structure. ${ }^{27,46}$ Their self-assembly is largely governed by London dispersion interactions. ${ }^{27}$ Accordingly, diamondoids can not only diffuse and rotate on the surface but may also appear to roll on it. Therefore, only recent advances in noncontact atomic force microscopy (nc-AFM) techniques using $\mathrm{CO}^{46-48}$ and $\mathrm{Cu}-\mathrm{O}$ terminated tips ${ }^{38,39}$ that provide submolecular resolution on the top atoms make it possible to confirm the bulky molecule rolling effect, which will vary the top atoms.

We considered and tested different metal surfaces $[\mathrm{Au}(111)$, $\mathrm{Ag}(111)$, and $\mathrm{Cu}(111)]$, and copper was identified as the best metal template due to its strongest confinement and because it is an excellent catalyst for on-surface reactions of 4,9diamantane dicarboxylic acid (DDA) (Figure S1). Because of hydrogen bonding between the carboxylic groups and interaction of the molecules with the surface, the predicted orientation of DDA while adsorbed on $\mathrm{Cu}(111)$ is shown in Figure $1 \mathrm{~b}$. Note that the distances between the carbon atoms of the carboxylic group and the metal surface $d_{1}$ and $d_{2}$ might be different and vary depending on the on-surface orientation of the diamantane scaffold. A proposed reaction pathway for stepwise on-surface annealing reactions is depicted in Figure 1c. Rotation of the carboxylic groups enables a contact between the surface and the corresponding atoms (first $\mathrm{H}$, then $\mathrm{O}$, and finally $\mathrm{C}$ ) and during the subsequent thermal treatment results in the induced surface-catalyzed dehydrogen-ation, followed by deoxygenation and finally the carbon-metal coordination processes takes place. Consequently, on-surface reaction products will have varying distances $d_{1}$ and $d_{2}$, and therefore the apparent rolling of diamantane framework will be induced, thus forming various orientations of DDA derivatives on $\mathrm{Cu}(111)$. 

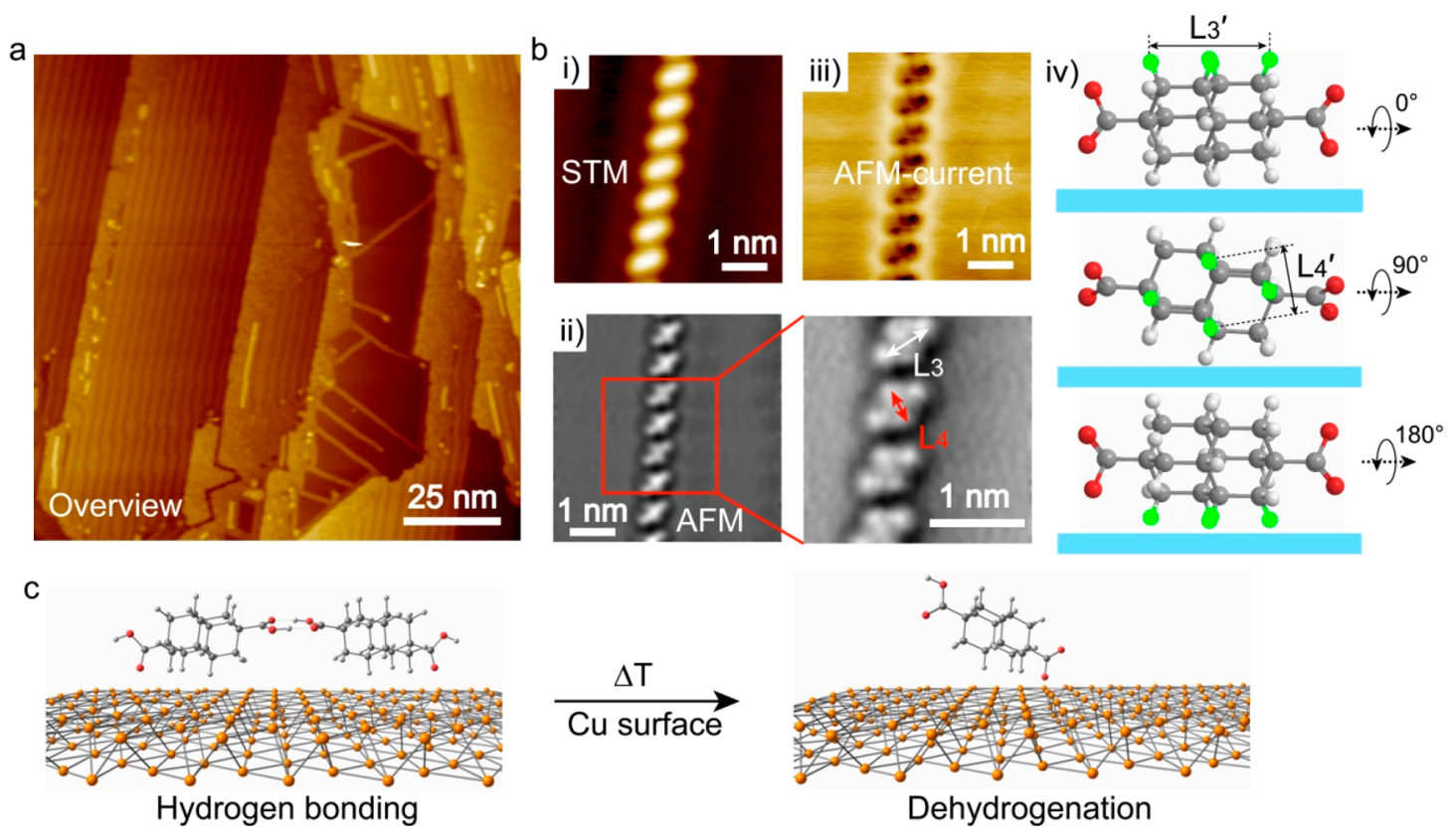

Figure 3. Formation of single and double diamantane chains on the $\mathrm{Cu}(111)$ surface. (a) An overview STM image $(125 \mathrm{~nm} \times 125 \mathrm{~nm})$. (b) Magnified STM image (i, -2 V, $10 \mathrm{pA}$ ) of single diamantane chains, as well as the corresponding nc-AFM constant height image (ii) and simultaneously current images (iii) at $A_{\text {osc }}=0.7 \AA, V=25 \mathrm{mV}$; here, their sizes are $5 \mathrm{~nm} \times 5 \mathrm{~nm}$, and (b-ii) zoomed-in nc-AFM image has dimensions of $2.4 \mathrm{~nm} \times 2.4 \mathrm{~nm}$. (b-iv) Top, the side view of bis-dehydrogenated DDA; middle, the side view of bis-dehydrogenated DDA after being rotated $90^{\circ}$ along the axis parallel to substrate surface; bottom, the side view of bis-dehydrogenated DDA after being rotated $180^{\circ}$ along the axis parallel to substrate surface. (c) Computed orientations of DDA molecules when engaging in hydrogen bonding and after the first step of dehydrogenation upon thermal treatment.

We showed previously that 2,6-naphthalenedicarboxylic acid self-assembles on metal surfaces via a process driven by carboxylic acid hydrogen bonding. ${ }^{34}$ However, in case of DDA, different modes of hydrogen-bonding interactions are feasible, head-to-tail, head-to-head, and tail-to-tail (Figure 2a). Figure 2b-i depicts two different hydrogen-bonding phases (I, head-totail and II, head-to-head/tail-to-tail) in DDA self-assemblies. Note that STM images of such high resolution were obtained by using a $\mathrm{Cu}-\mathrm{O}$ terminated tip. ${ }^{38,39}$ Upon inspection of the images, one notices that DDA molecules show slightly different heights in contrast even in the same phase (for example, the DDA molecule in the central black rectangle shows higher brightness in spatial distribution than the other two DDAs in neighbored black rectangles), as marked by the black and red rectangles (Figure $2 \mathrm{~b}$ ). This may be caused by the rotational flexibility of carboxylic hydrogen bonding versus $\mathrm{Cu}$ surface to finely tune its height (hydrogen bonding plane parallel or vertical versus $\mathrm{Cu}$ surface). Furthermore, the hydrogen-bonding alignment direction marked by a black dashed line in phase I is different from that in phase II (the red line in Figure $2 \mathrm{~b}$ ). Thus, the self-assembly structure of DDA is not a fully periodic structure like that of a 3D crystal. However, for two DDA molecules engaging in both hydrogen-bonding phases, it was found that the distances $D_{1}(\mathrm{COOH}-\mathrm{COOH})$ and $D_{2}(\mathrm{COOH}$ $-\mathrm{COOH})$ were both $1.88 \pm 0.01 \mathrm{~nm}$, agreeing with the computed distance of $1.88 \mathrm{~nm}$ very well (Figure S9). It has been demonstrated that high-resolution nc-AFM imaging with a CO-modified tip is capable of showing the terminal hydrogens of bulky molecules on surfaces. ${ }^{27,46}$ To reduce effects due to tip flexibility, we used the more rigid $\mathrm{Cu}-\mathrm{O}$ tip termination for the present study. ${ }^{39}$ Accordingly, the top hydrogens could clearly be visualized and identified (Figure 2c-ii and d-ii), where the corresponding structural assignments were further confirmed by the simultaneous recorded STM images (Figure $2 \mathrm{c}-\mathrm{i}$ and $\mathrm{d}-\mathrm{i}$ ). These results strongly suggest that the main adsorption configurations of DDA are different in phases I and II, because different top hydrogens are pointing in the perpendicular direction from the metal surface. Even inside phase I, it is noticeable that hydrogen heights, as determined from image brightness, are different in Figure $2 c-i$ and $c$-ii, respectively, suggesting that the configurations are slightly different. Note that this could not be observed only using the STM images (compare Figure $2 \mathrm{~d}$-i with $\mathrm{d}$-ii), once again confirming the complementarity of STM and AFM imaging techniques when trying to discern the on-surface assembly and behavior of bulky $3 \mathrm{D}$ molecules. As shown in Figure $2 \mathrm{c}$-iii and d-iii, both proposed DDA configurations, with one side carboxylic group higher and the other side lower relatively to substrate surface, show very good agreement in nc-AFM contrast signatures and atom-to-atom distances (ex-perimental values, $L_{1}, 4.72 \pm 0.1 \AA$ and $L_{2}, 2.88 \pm 0.1 \AA$; computed distances, $L_{1}{ }^{\prime}, 4.87 \AA$ and $L_{2}{ }^{\prime}, 2.56 \AA$ ). Note a mismatch between the DDA nc-AFM image feature (Figure 2c-ii) of phase I and the proposed configuration of Figure 2c-iii; it is presumably caused by slight tilting/rolling adjusted by the hydrogen bonding with neighboring DDA molecules. This indicates that our nc-AFM data are governed by the top hydrogen atoms of $3 \mathrm{D}$ molecules, even though two hydrogen atoms at the same carbon cannot be distinguished clearly enough (Figure 2c-ii and c-iii). Additionally, we found evidence that the nc-AFM tip can roll the DDA molecules during scanning, thus resulting in a new configuration (Figure S2).

Formation of Single and Double Diamantane Chains. The subsequent thermal treatment $\left(\sim 157{ }^{\circ} \mathrm{C}\right)$ triggered dehydrogenation and deoxygenation reactions (Figure S3), 
resulting in DDA-derived single and double chains (Figure 3a). Figure 3b-i depicts a magnified STM image of the single chains that is markedly different from the self-assembly structure, shown in Figure 2. Dehydrogenation of carboxylic groups most probably causes the molecule to rotate and bind directly to the metal surface via a $\mathrm{COO}-\mathrm{Cu}$ bond (Figure $3 \mathrm{c}$ ). Furthermore, it is deduced that the dehydrogenation occurred on both DDA carboxylic groups of the single chains because they show the same contrast in STM and nc-AFM measurements (Figure 3b-i and b-ii). The breaking of the hydrogen-bond network is evident from a new configuration of the diamantane cage that was confirmed by the recorded nc-AFM image (Figure 3b-ii). This new configuration appears to be quite similar to the configuration induced by the scanning tip (Figure S2). A spatial bis-dehydrogenated DDA configuration is further demonstrated by Figure 3b-iv, with the top hydrogen atoms (relatively flat in a plane parallel to the substrate surface) satisfying the nc-AFM image feature and also a satisfactory atom-to-atom distance agreement (experimental value, $L_{3}, 4.88$ $\pm 0.1 \AA$ and $L_{4}, 2.95 \pm 0.1 \AA$; computed distances, $L_{3}{ }^{\prime}, 4.42 \AA$ and $L_{4}{ }^{\prime}, 2.48 \AA$ ). Note that a reasonable large error between experimental and theoretical values was obtained on the two indistinguishable hydrogen atoms of the $\mathrm{DDA} \mathrm{CH} \mathrm{CH}_{2}$ groups.

The simultaneously recorded AFM current image (Figure $3 \mathrm{~b}$ iii) can be interpreted as a conductivity map of DDA assemblies on the metal surface. Note that the right and left sides of the single chains show higher conductivity (they are brighter) than the metal site, probably caused by the carboxylate slightly pulling the $\mathrm{Cu}$ atoms off the surface (shorter distance to the STM tip causes higher tunnelling current). These results agree well with the conjecture of carboxylic bonding to the metal surface, implying enhanced conductivity of the carboxylic part. Furthermore, STM manipulations of such single chains support the $\mathrm{COO}-\mathrm{Cu}$ bonding hypothesis and not covalent interactions between diamantane scaffolds (Figure S4). In addition to straight single chains, curved single chains were also identified and resulted in nc-AFM topology and AFM current images comparable to the images of the straight chains (Figure S5).

A further type of product that we observed are DDA derived double chains. Figure 4a-i and -ii depicts magnified STM and ncAFM images of a straight double chain, with one single chain on each side. Note that the metal atoms inside the double chains could be clearly identified as the dots in the AFM current image marked by a black dashed rectangle (Figure 4a-iii) and the periodic distance of $\mathrm{Cu}$ atoms was measured as $0.67 \pm 0.01 \mathrm{~nm}$. On the basis of this, we can assume that two single chains were further chemically linked by a set of common $\mathrm{Cu}$ atoms via a deoxygenation process (Figure 4c). Such reaction further pulls single copper atoms up from the surface and consequently tilts the diamantane framework because the distance between the carboxylic carbon atom and the surface shortens. This tiny tilt (labeled $\Theta$ ) i s further confirmed by the proposed spatial configuration in Figure 4b-iv, which can also be supported by the features of nc-AFM image on double chains (the hydrogen atom near the $\mathrm{Cu}$ linkage is obviously higher than the other hydrogen atoms). Note that computational geometry optimization provides three stable positions for such $\mathrm{Cu}$ atoms sitting above, below, and in the molecular plane (Figure S7). However, the inplane case can be excluded by XPS analysis because the carboxylic $\mathrm{O}$ is peak corresponding to the $(\mathrm{C}=\mathrm{O})-\mathrm{Cu}$ component is kept unchanged after dehydrogenation, and mainly the carboxylic C

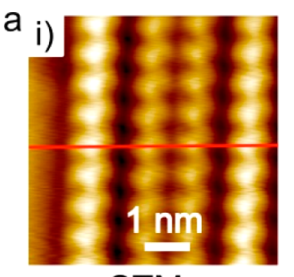

STM

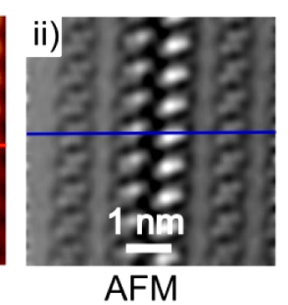

AFM
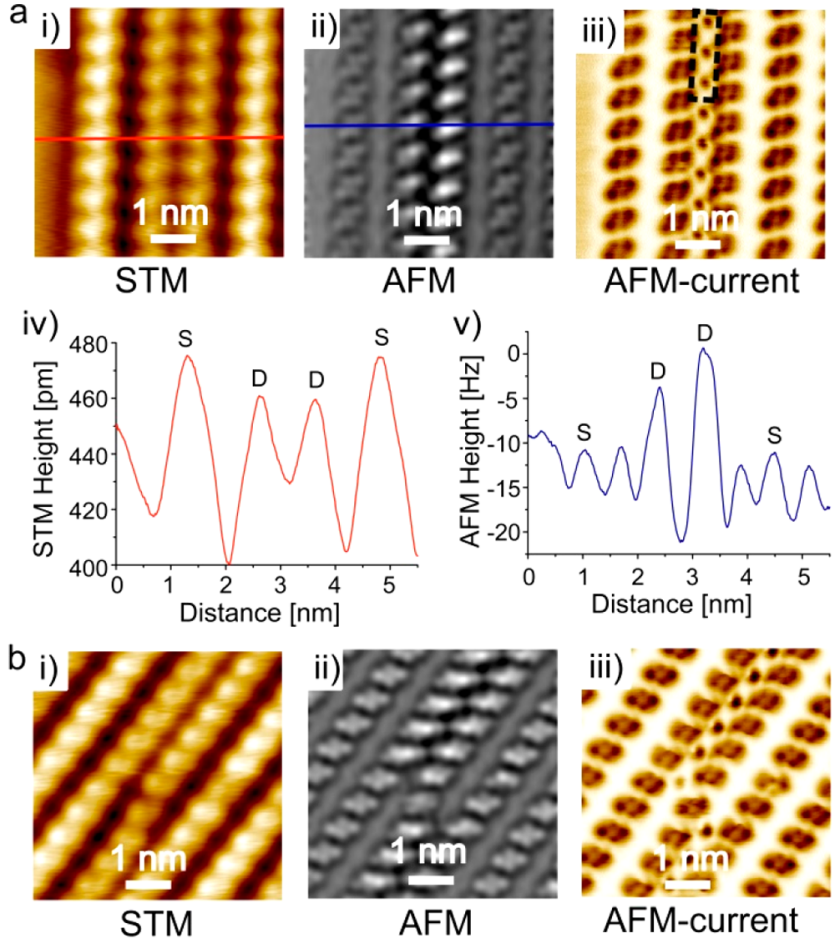

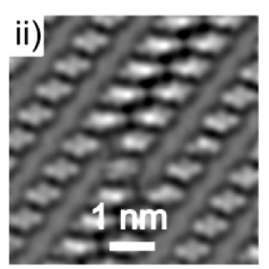

AFM

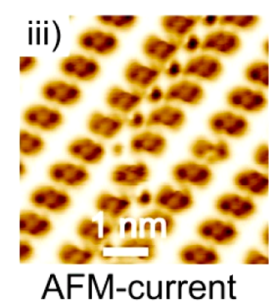

AFM-current iv)
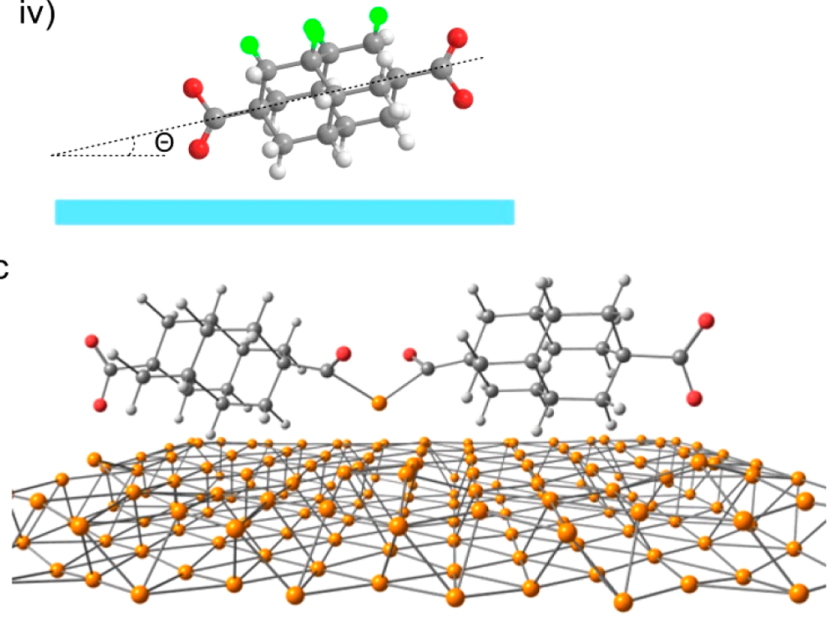

Figure 4. Formation of double DDA chains on the $\mathrm{Cu}(111)$ surface. ( $a$ and b) Magnified STM images (i, $-2 \mathrm{~V}, 10 \mathrm{pA}$ ), nc-AFM images (ii), and simultaneous nc-AFM current images (iii) at $A_{\text {osc }}=0.7 \AA, V$ $=25 \mathrm{mV}$ of straight double DDA chains $(\mathrm{a}, 5.5 \mathrm{~nm} \times 5.5 \mathrm{~nm})$ and splitting double DDA chains (b, $5.5 \mathrm{~nm} \times 5.5 \mathrm{~nm})$; a-iv and a-v are the corresponding line profiles as marked with a red line in a-i and blue line in a-ii, respectively; b-iv is the side view of the proposed tiny tilt bis-dehydrogenated DDA. (c) Computed orientation of two dehydroxylated DDA molecules forming double chains with a copper atom in the middle (central $\mathrm{Cu}$ atom below the molecular plane).

1s peak is shifting to lower energy; for more details, see Figure 5f. The above-plane case is also unlikely; otherwise, our ncAFM tip should detect such metal atoms due to their size. ${ }^{49}$ What is more, double chains appear with a lower intensity than single chains in the STM image, whereas they appear topographically higher as suggested by the nc-AFM data. For better comparison, we also drew two line profiles across the single and double chains, as marked by the red and blue lines (Figure 4a-iv and v). The obtained results indicate that double chains essentially form a nanowire because they exhibit higher conductivity (lower contrast in STM image) than single chains 

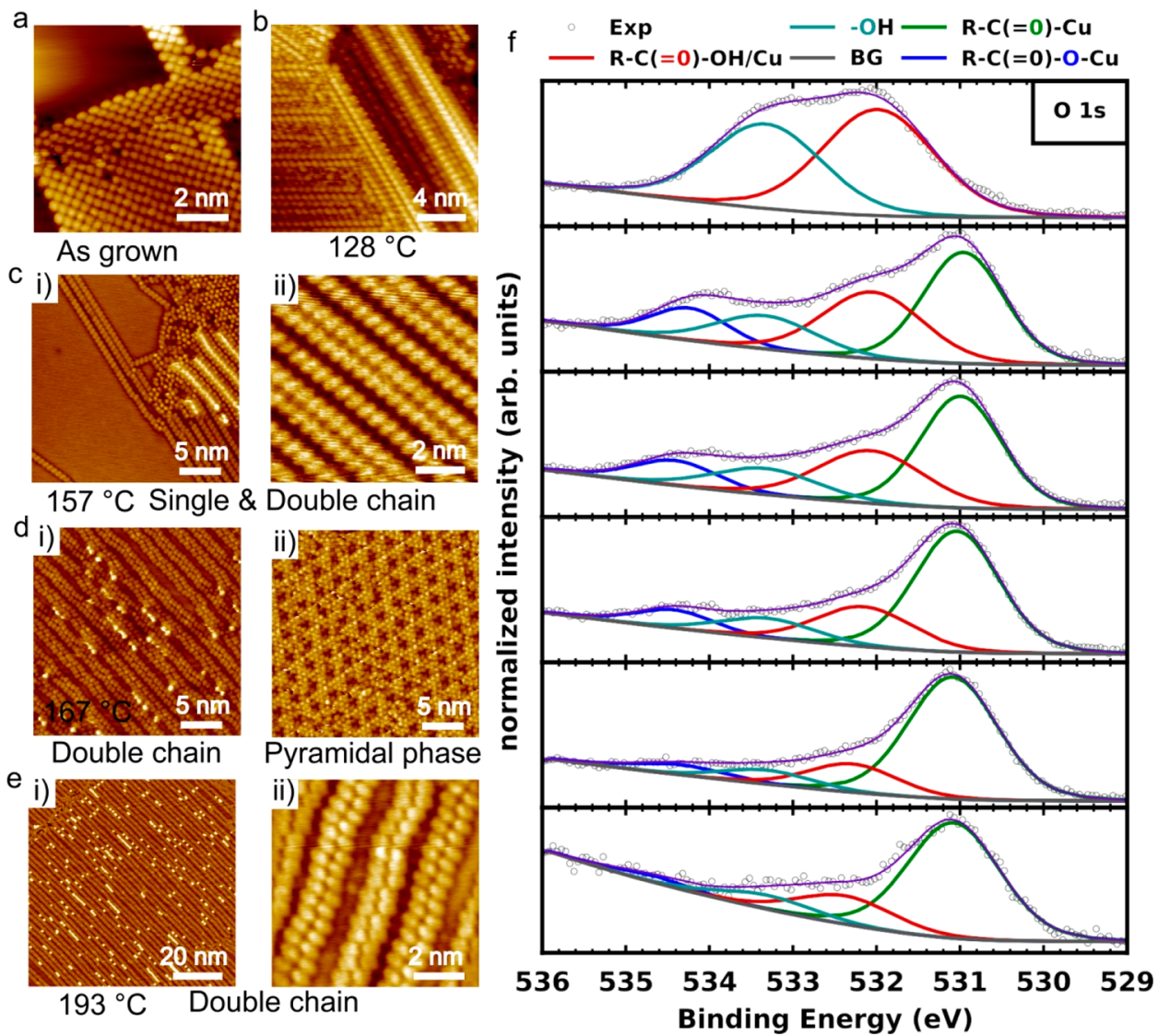

Figure 5. XPS characterization of DDA derivatives on a $\mathrm{Cu}(111)$ surface. (a-e) Representative STM images after multiannealing steps: as-grown $(-0.5 \mathrm{~V}, 10 \mathrm{pA}, 8 \times 8 \mathrm{~nm}), 128^{\circ} \mathrm{C}(1.3 \mathrm{~V}, 10 \mathrm{pA}, 17 \times 17 \mathrm{~nm}), 157^{\circ} \mathrm{C}(\mathrm{i},-1 \mathrm{~V}, 10 \mathrm{pA}, 25 \times 25 \mathrm{~nm}$ and ii, $-0.5 \mathrm{~V}, 5 \mathrm{pA}, 8.4 \times 8.4 \mathrm{~nm}), 167^{\circ} \mathrm{C}(\mathrm{i}$, $-1 \mathrm{~V}$, and ii, $-0.5 \mathrm{~V}$, both at $10 \mathrm{pA}, 25 \times 25 \mathrm{~nm}$ ), and $193{ }^{\circ} \mathrm{C}$ (i, overview, $-1 \mathrm{~V}, 10 \mathrm{pA}, 84 \times 84 \mathrm{~nm}$, and ii, zoomed-in, $-0.3 \mathrm{~V}, 100 \mathrm{pA}, 8.2 \times 8.2$ $\mathrm{nm}$ ). (f) High-resolution XPS spectra on $\mathrm{O} 1 \mathrm{~s}$ core levels of DDA derived sample from as-grown (top) to 5 steps annealing temperatures (bottom, the final step corresponds to annealing at $245{ }^{\circ} \mathrm{C}$ ).

due to the insertion of copper, while in the AFM images double chains appear topographically higher than the single chains due to the tilted orientation of the diamantane cage.

We also observed the splitting effect of a double chain as revealed by imaging a single chain that proceeded to form double chains with the adjacent two single chains (Figure 4b-i). The two diamondoid molecules located at switching positions again show different on-surface orientations of the assemblies (Figure 4b-ii). As expected, the two lines of the linked $\mathrm{Cu}$ atoms in such splitting double chains could be clearly observed (Figure 4b-iii).

By carefully adjusting the thermal treatment, the reaction output can be controlled and optimized (Figure 5a-e). Gentle annealing (below $128{ }^{\circ} \mathrm{C}$ ) mainly broke the self-assembled structure and induced the formation of structures with a higher roughness (not a flat monolayer structure) (Figure $5 b$ ). Single and double chain structures appeared after annealing at around $157{ }^{\circ} \mathrm{C}$. However, disordered DDA-derived assembly structures still coexisted, and we also identified top chains sitting on single chains (Figure $5 \mathrm{c}-\mathrm{i}$ ). By increasing the annealing temperature to $167^{\circ} \mathrm{C}$, more double chains formed (Figure 5di), and a supra-structure of the pyramidal phase was also observed (Figures 5d-ii and S6). Upon further increase of the annealing temperature to $193{ }^{\circ} \mathrm{C}$, DDA derived double chains formed as the main product in high quality and at large scale (Figure 5e), with no single chains and only a few molecules in a disordered phase (Figure S3E). Ultimately, annealing above
$203{ }^{\circ} \mathrm{C}$ destroyed the double chains and formed disordered unidentified species (Figure S3).

XPS Characterization of DDA Derivatives. To further explore and characterize the observed on-surface chemistry of DDA, XPS measurements were performed on the samples obtained from different temperature annealing protocols (Figure 5). We found that the $\mathrm{C} 1 \mathrm{~s}$ main peaks corresponding to carbons of the diamantane cage do not change during the various annealing processes. However, the carboxylic $\mathrm{C}$ 1s main peak undergoes pronounced shifts from the as-grown sample $(288.95 \mathrm{eV})$ to the double chains as the main product (287.61 eV) (Figure S7 and Table S1). This is further supported by an analysis of the $\mathrm{O} 1 \mathrm{~s}$ core level data, which also shows pronounced changes and allows tracking of the chemical interconversion of DDA derivatives. On that note, Figure $5 \mathrm{f}$ shows two shifted peaks at 531.04 and $534.39 \mathrm{eV}$, corresponding to $-\mathrm{C}(=\underline{\mathrm{O}})-\mathrm{Cu}$ and $-\mathrm{C}(=\mathrm{O})-\mathrm{O}-\mathrm{Cu}$, respectively (detailed in Table S2). The appearance of the $\mathrm{O} 1 \mathrm{~s}$ peak for the $-\mathrm{C}(=\mathrm{O})-\mathrm{O}-\mathrm{Cu}$ moiety strongly points toward a dehydrogenation reaction, and then it was gradually reduced further, as evident by comparison with the $\mathrm{O}$ 1s peak of $-\mathrm{C}(=$ $\mathrm{O})-\mathrm{Cu}$. These data confirm the subsequent deoxygenation reaction. Thus, we deduce that, as dehydrogen-ation occurs, the hydroxyl oxygen atom of the carboxylic group cannot reach the $\mathrm{Cu}(111)$ surface due to a spatial restriction (the chemical $\mathrm{Cu}$ $-\mathrm{O}$ bonding would be elongated and weakened), and the $\mathrm{O} 1 \mathrm{~s}$ peak of such radical-like oxygen is 
therefore shifted to higher binding energies when compared to its initial peak at $533.32 \mathrm{eV}$. Similarly, as deoxygenation occurs, the bonding of the derivative with a carbonyl $-\mathrm{C}(=\mathrm{O})-\mathrm{Cu}-$ $\mathrm{C}(=\mathrm{O})$ will occur, and its $\mathrm{O} 1 \mathrm{~s}$ peak is shifted to lower binding energies when compared to its initial peak at 531.98 $\mathrm{eV}$. We also observed that the intensity of the $\mathrm{O} 1 \mathrm{~s}$ double peak corresponding to the intact carboxylic group was continuously reduced during annealing.

To support our assignments of the observed species, we performed gas-phase DFT computations to estimate the chemical shifts of the initial and final states (Figure S8). Our results are in qualitative agreement with the measured values, showing that the oxygen signal of the $-\mathrm{C}(=\mathrm{O})-\mathrm{Cu}-\mathrm{C}(=\mathrm{O})$ - species is considerably shifted to lower binding energies (Table S3) upon metalation of the carbon atom. This further confirms the proposed on-surface reaction. Additionally, to confirm the proposed reactivity of DDA molecules upon annealing and metallic chain formation, we performed a theoretical study using the semiempirical GFN-xTB computational approach. ${ }^{47}$ All details are provided in Figures S9-S11 and Tables S4-S6. We found an overall excellent match between the computed geometries of DDA structures on the $\mathrm{Cu}(111)$ surface and the experimentally observed data.

\section{CONCLUSION}

Surface-controlled dehydrogenation and deoxygenation processes of carboxylic groups provide a reproducible route to tailor the anchoring of diamondoids to metal surfaces by building higher-order single diamondoid-chains from the lower-order chains. Such rigorous spatial control enables manufacturing of one atom wide metallic chains, a feat with important implications for nanotechnology and electronic device down-sizing. This strategy provides opportunities for analyzing physical properties of such quantum-scale electronic circuitry, for example, their band structure, magnetism, and charge/spin transport along the set of spatial periodic magnetic metal atoms, etc. Furthermore, the size of the anchoring layer can be readily adjusted because diamondoids (as well as other well-defined and readily available spacers) come in different shapes and sizes, and thus provide additional control and enrich the number of possible ensembles for on-surface architectures capable of charge/spin transport.

\section{The Supporting Information:}

Additional data and analysis by STM, nc-AFM, XPS, and computations.

\section{Corresponding Authors \\ *gaoh@uni-muenster.de \\ *prs@uni-giessen.de \\ *fuchsh@uni-muenster.de}

\section{Notes}

The authors declare no competing financial interest.

\section{ACKNOWLEDGMENTS}

We thank Prof. Ferdi Schüth for discussions. This work was financially supported by the Deutsche Forschungsgemeinschaft (DFG): TRR 61, SFB 858, GA 2430/1-1, MO 2345/4-1, and Schr 597/27-2 (Priority Program "Dispersion”, SPP1807). M.S. thanks the Alexander-von-Humboldt Foundation for a Return Fellowship. Computations were conducted on the HALO high-performance computer at the Justus Liebig University, Giessen, Germany.

\section{REFERENCES}

(1) Xiang, D.; Wang, X.; Jia, C.; Lee, T.; Guo, X. Molecular-Scale Electronics: From Concept to Function. Chem. Rev. 2016, 116, 43184440.

(2) Su, T. A.; Neupane, M.; Steigerwald, M. L.; Venkataraman, L.; Nuckolls, C. Chemical principles of single-molecule electronics. Nat. Rev. Mater. 2016, 1, 16002.

(3) Vilan, A.; Aswal, D.; Cahen, D. Large-Area, Ensemble Molecular Electronics: Motivation and Challenges. Chem. Rev. 2017, 117, 42484286.

(4) Sanvito, S. Molecular spintronics. Chem. Soc. Rev. 2011, 40, 3336-3355.

(5) Shiraishi, M.; Ikoma, T. Molecular spintronics. Phys. E (Amsterdam, Neth.) 2011, 43, 1295-1317.

(6) O'Mullane, A. P. From single crystal surfaces to single atoms: investigating active sites in electrocatalysis. Nanoscale 2014, 6, 40124026.

(7) Ling, C.; Shi, L.; Ouyang, Y.; Zeng, X. C.; Wang, J. Nanosheet Supported Single-Metal Atom Bifunctional Catalyst for Overall Water Splitting. Nano Lett. 2017, 17, 5133-5139.

(8) Metzger, R. M. Unimolecular Electrical Rectifiers. Chem. Rev. 2003, 103, 3803-3834.

(9) Metzger, R. M. Unimolecular rectifiers: Present Status. Chem. Phys. 2006, 326, 176-187.

(10) Randel, J. C.; Niestemski, F. C.; Botello-Mendez, A. R.; Mar, W.; Ndabashimiye, G.; Melinte, S.; Dahl, J. E. P.; Carlson, R. M. K.; Butova, E. D.; Fokin, A. A.; Schreiner, P. R.; Charlier, J.-C.; Manoharan, H. C. Unconventional molecule-resolved current rectification in diamondoid-fullerene hybrids. Nat. Commun. 2014, 5, 4877.

(11) Bowler, D. R. Atomic-scale nanowires: physical and electronic structure. J. Phys.: Condens. Matter 2004, 16, R721.

(12) Agrait, N.; Yeyati, A. L.; van Ruitenbeek, J. M. Quantum properties of atomic-sized conductors. Phys. Rep. 2003, 377, 81-279.

(13) Ohnishi, H.; Kondo, Y.; Takayanagi, K. Quantized conductance through individual rows of suspended gold atoms. Nature 1998, 395, $780-783$.

(14) Yanson, A. I.; Bollinger, G. R.; van den Brom, H. E.; Agrait, N.; van Ruitenbeek, J. M. Formation and manipulation of a metallic wire of single gold atoms. Nature 1998, 395, 783-785.

(15) Nilius, N.; Wallis, T. M.; Ho, W. Development of OneDimensional Band Structure in Artifical Gold Chains. Science 2002, 297, 1853-1856.

(16) Nilius, N.; Wallis, T. M.; Persson, M.; Ho, W. Interplay between Electronic Properties and Interatomic Spacing in Artificial Gold Chains on $\mathrm{NiAl}(110)$. J. Phys. Chem. C 2014, 118, 2900129006.

(17) González, J. C.; Rodrigues, V.; Bettini, J.; Rego, L. G. C.; Rocha, A. R.; Coura, P. Z.; Dantas, S. O.; Sato, F.; Galvão, D. S.; Ugarte, D. Indication of Unusual Pentagonal Structures in AtomicSize Cu Nanowires. Phys. Rev. Lett. 2004, 93, 126103.

(18) Guo, H.; Lin, N.; Chen, Y.; Wang, Z.; Xie, Q.; Zheng, T.; Gao, N.; Li, S.; Kang, J.; Cai, D.; Peng, D.-L. Copper Nanowires as Fully Transparent Conductive Electrodes. Sci. Rep. 2013, 3, 2323. 
(19) Chang, Y.; Lye, M. L.; Zeng, H. C. Large-Scale Synthesis of High-Quality Ultralong Copper Nanowires. Langmuir 2005, 21, 3746-3748.

(20) Mendoza-Cruz, R.; Bazán-Díaz, L.; Velázquez-Salazar, J. J.; Plascencia-Villa, G.; Bahena-Uribe, D.; Reyes-Gasga, J.; Romeu, D.; Guisbiers, G.; Herrera-Becerra, R.; José-Yacamán, M. Helical Growth of Ultrathin Gold-Copper Nanowires. Nano Lett. 2016, 16, 15681573.

(21) Ye, S.; Stewart, I. E.; Chen, Z.; Li, B.; Rathmell, A. R.; Wiley, B. J. How Copper Nanowires Grow and How To Control Their Properties. Acc. Chem. Res. 2016, 49, 442-451.

(22) Ai, Y.; Smida, H.; Ghilane, J.; Vilà, N.; Ghanbaja, J.; Walcarius, A.; Lacroix, J. C. Copper Nanowires through Oriented Mesoporous Silica: A Step towards Protected and Parallel Atomic Switches. Sci. Rep. 2017, 7, 17752.

(23) Chen, H. M.; Liu, R.-S. Architecture of Metallic Nanostructures: Synthesis Strategy and Specific Applications. J. Phys. Chem. C 2011, 115, 3513-3527.

(24) Schwertfeger, H.; Fokin, A. A.; Schreiner, P. R. Diamonds are a Chemist's Best Friend: Diamondoid Chemistry Beyond Adamantane. Angew. Chem., Int. Ed. 2008, 47, 1022-1036.

(25) Dahl, J. E.; Liu, S. G.; Carlson, R. M. K. Isolation and Structure of Higher Diamondoids, Nanometer-Sized Diamond Molecules. Science 2003, 299, 96-99.

(26) Gunawan, M. A.; Hierso, J.-C.; Poinsot, D.; Fokin, A. A.; Fokina, N. A.; Tkachenko, B. A.; Schreiner, P. R. Diamondoids: functionalization and subsequent applications of perfectly defined molecular cage hydrocarbons. New J. Chem. 2014, 38, 28-41.

(27) Ebeling, D.; Šekutor, M.; Stiefermann, M.; Tschakert, J.; Dahl, J. E. P.; Carlson, R. M. K.; Schirmeisen, A.; Schreiner, P. R. London Dispersion Directs On-Surface Self-Assembly of [121]Tetramantane Molecules. ACS Nano 2017, 11, 9459-9466.

(28) Yang, W. L.; Fabbri, J. D.; Willey, T. M.; Lee, J. R. I.; Dahl, J. E.; Carlson, R. M. K.; Schreiner, P. R.; Fokin, A. A.; Tkachenko, B. A.; Fokina, N. A.; Meevasana, W.; Mannella, N.; Tanaka, K.; Zhou, X. J.; van Buuren, T.; Kelly, M. A.; Hussain, Z.; Melosh, N. A.; Shen, Z.-X. Monochromatic Electron Photoemission from Diamondoid Monolayers. Science 2007, 316, 1460-1462.

(29) Zhang, J.; Zhu, Z.; Feng, Y.; Ishiwata, H.; Miyata, Y.; Kitaura, R.; Dahl, J. E. P.; Carlson, R. M. K.; Fokina, N. A.; Schreiner, P. R.; Tománek, D.; Shinohara, H. Evidence of Diamond Nanowires Formed inside Carbon Nanotubes from Diamantane Dicarboxylic Acid. Angew. Chem., Int. Ed. 2013, 52, 3717-3721.

(30) Nakanishi, Y.; Omachi, H.; Fokina, N. A.; Schreiner, P. R.; Kitaura, R.; Dahl, J. E. P.; Carlson, R. M. K.; Shinohara, H. Template Synthesis of Linear-Chain Nanodiamonds Inside Carbon Nanotubes from Bridgehead-Halogenated Diamantane Precursors. Angew. Chem., Int. Ed. 2015, 54, 10802-10806.

(31) Narasimha, K. T.; Ge, C.; Fabbri, J. D.; Clay, W.; Tkachenko, B. A.; Fokin, A. A.; Schreiner, P. R.; Dahl, J. E.; Carlson, R. M. K.; Shen, Z. X.; Melosh, N. A. Ultralow effective work function surfaces using diamondoid monolayers. Nat. Nanotechnol. 2016, 11, 267-272.

(32) Yan, H.; Hohman, J. N.; Li, F. H.; Jia, C.; Solis-Ibarra, D.; Wu, B.; Dahl, J. E. P.; Carlson, R. M. K.; Tkachenko, B. A.; Fokin, A. A.; Schreiner, P. R.; Vailionis, A.; Kim, T. R.; Devereaux, T. P.; Shen, Z. - X.; Melosh, N. A. Hybrid metal-organic chalcogenide nanowires with electrically conductive inorganic core through diamondoid directed assembly. Nat. Mater. 2017, 16, 349-355.

(33) Fokina, N. A.; Tkachenko, B. A.; Dahl, J. E. P.; Carlson, R. M. K.; Fokin, A. A.; Schreiner, P. R. Synthesis of Diamondoid Carboxylic Acids. Synthesis 2012, 44, 259-264.

(34) Gao, H.-Y.; Held, P. A.; Knor, M.; Mück-Lichtenfeld, C.; Neugebauer, J.; Studer, A.; Fuchs, H. Decarboxylative Polymerization of 2,6-Naphthalenedicarboxylic Acid at Surfaces. J. Am. Chem. Soc. 2014, 136, 9658-9663.

(35) Shen, Q.; Gao, H.-Y.; Fuchs, H. Frontiers of on-surface synthesis: From principles to applications. Nano Today 2017, 13, 7796.
(36) Held, P. A.; Gao, H. Y.; Liu, L.; Mück-Lichtenfeld, C.; Timmer, A.; Mönig, H.; Barton, D.; Neugebauer, J.; Fuchs, H.; Studer, A. OnSurface Domino Reactions: Glaser Coupling and Dehydrogenative Coupling of a Biscarboxylic Acid To Form Polymeric Bisacylperoxides. Angew. Chem., Int. Ed. 2016, 55, 9777-9782.

(37) Gao, H.-Y.; Held, P. A.; Amirjalayer, S.; Liu, L.; Timmer, A.; Schirmer, B.; Díaz Arado, O.; Mönig, H.; Mück-Lichtenfeld, C.; Neugebauer, J.; Studer, A.; Fuchs, H. Intermolecular On-Surface $\sigma$ Bond Metathesis. J. Am. Chem. Soc. 2017, 139, 7012-7019.

(38) Mönig, H.; Hermoso, D. R.; Díaz Arado, O.; Todorović, M.; Timmer, A.; Schüer, S.; Langewisch, G.; Pérez, R.; Fuchs, H. Submolecular Imaging by Noncontact Atomic Force Microscopy with an Oxygen Atom Rigidly Connected to a Metallic Probe. ACS Nano 2016, 10, 1201-1209.

(39) Mönig, H.; Amirjalayer, S.; Timmer, A.; Hu, Z.; Liu, L.; Diaz Arado, O.; Cnudde, M.; Strassert, C. A.; Ji, W.; Rohlfing, M.; Fuchs, $\mathrm{H}$. Quantitative assessment of intermolecular interactions by atomic force microscopy imaging using copper oxide tips. Nat. Nanotechnol. 2018, 13, 371-375.

(40) Becke, A. D. Density-functional thermochemistry. III. The role of exact exchange. J. Chem. Phys. 1993, 98, 5648-5652.

(41) Lee, C.; Yang, W.; Parr, R. G. Development of the ColleSalvetti correlation-energy formula into a functional of the electron density. Phys. Rev. B: Condens. Matter Mater. Phys. 1988, 37, 785-789.

(42) Grimme, S.; Antony, J.; Ehrlich, S.; Krieg, H. A consistent and accurate $a b$ initio parametrization of density functional dispersion correction (DFT-D) for the 94 elements H-Pu. J. Chem. Phys. 2010, 132,154104

(43) Grimme, S.; Ehrlich, S.; Goerigk, L. Effect of the damping function in dispersion corrected density functional theory. J. Comput. Chem. 2011, 32, 1456-1465.

(44) Frisch, M. J.; Trucks, G. W.; Schlegel, H. B.; Scuseria, G. E.; Robb, M. A.; Cheeseman, J. R.; Scalmani, G.; Barone, V.; Petersson, G. A.; Nakatsuji, H.; Li, X.; Caricato, M.; Marenich, A. V.; Bloino, J.; Janesko, B. G.; Gomperts, R.; Mennucci, B.; Hratchian, H. P.; Ortiz, J. V.; Izmaylov, A. F.; Sonnenberg, J. L.; Williams-Young, D.; Ding, F.; Lipparini, F.; Egidi, F.; Goings, J.; Peng, B.; Petrone, A.; Henderson, T.; Ranasinghe, D.; Zakrzewski, V. G.; Gao, J.; Rega, N.; Zheng, G.; Liang, W.; Hada, M.; Ehara, M.; Toyota, K.; Fukuda, R.; Hasegawa, J.; Ishida, M.; Nakajima, T.; Honda, Y.; Kitao, O.; Nakai, H.; Vreven, T.; Throssell, K.; Montgomery, J. A., Jr.; Peralta, J. E.; Ogliaro, F.; Bearpark, M. J.; Heyd, J. J.; Brothers, E. N.; Kudin, K. N.; Staroverov, V. N.; Keith, T. A.; Kobayashi, R.; Normand, J.; Raghavachari, K.; Rendell, A. P.; Burant, J. C.; Iyengar, S. S.; Tomasi, J.; Cossi, M.; Millam, J. M.; Klene, M.; Adamo, C.; Cammi, R.; Ochterski, J. W.; Martin, R. L.; Morokuma, K.; Farkas, O.; Foresman, J. B.; Fox, D. J. Gaussian 16, revision B.01; Gaussian, Inc.: Wallingford, CT, 2016.

(45) Grimme, S.; Bannwarth, C.; Shushkov, P. A Robust and Accurate Tight-Binding Quantum Chemical Method for Structures, Vibrational Frequencies, and Noncovalent Interactions of Large Molecular Systems Parametrized for All spd-Block Elements ( $\mathrm{Z}=1-$ 86). J. Chem. Theory Comput. 2017, 13, 1989-2009.

(46) Ebeling, D.; Sekutor, M.; Stiefermann, M.; Tschakert, J.; Dahl, J. E. P.; Carlson, R. M. K.; Schirmeisen, A.; Schreiner, P. R. Assigning the absolute configuration of single aliphatic molecules by visual inspection. Nat. Commun. 2018, 9, 2420.

(47) Gross, L.; Mohn, F.; Moll, N.; Liljeroth, P.; Meyer, G. The Chemical Structure of a Molecule Resolved by Atomic Force Microscopy. Science 2009, 325, 1110-1114.

(48) de Oteyza, D. G.; Gorman, P.; Chen, Y.-C.; Wickenburg, S.; Riss, A.; Mowbray, D. J.; Etkin, G.; Pedramrazi, Z.; Tsai, H.-Z.; Rubio, A.; Crommie, M. F.; Fischer, F. R. Direct Imaging of Covalent Bond Structure in Single-Molecule Chemical Reactions. Science 2013, 340, 1434-1437.

(49) Liu, L.; Klaasen, H.; Timmer, A.; Gao, H. Y.; Barton, D.; Mönig, H.; Neugebauer, J.; Fuchs, H.; Studer, A. $\alpha$-Diazo Ketones in On-Surface Chemistry. J. Am. Chem. Soc. 2018, 140, 6000-6005. 